\title{
Caracterización no lineal con excitación a ráfagas de transductores de potencia con piezocomposites 1-3 para emisión en aire
}

\author{
J. A. CASALS ${ }^{1}$, A. ALBAREDA ${ }^{1}$, R. PÉREZ ${ }^{1}$ Y F. MONTERO DE ESPINOSA ${ }^{2}$ \\ ${ }^{1}$ Dept. Física Aplicada. Univ. Politècnica Catalunya 08034 Barcelona. \\ ${ }^{2}$ Inst. Acústica. CSIC 28006 Madrid.
}

\begin{abstract}
En Ensayos No Destructivos, así como en la emisión de ultrasonidos, se utilizan frecuentemente transductores resonantes realizados con piezocomposites 1-3. Cuando el transductor piezocomposite se emplea emitiendo ultrasonidos en aire, es necesario el uso de pulsos de gran amplitud para generar una señal acústica elevada y tener en cuenta el régimen no lineal. Las medidas de caracterización no lineal se han llevado a cabo con excitación a ráfagas en condiciones próximas a las de las aplicaciones. Se propone un nuevo método que mide la corriente y la velocidad de vibración del transductor piezocomposite con una señal de excitación a ráfagas para frecuencias cercanas a la de resonancia grosor. La corriente de la respuesta estacionaria del transductor se mide justo antes del final de la ráfaga para diferentes composites. El uso de estas excitaciones nos permite medir el comportamiento no lineal de piezocomposites evitando el sobrecalentamiento de los transductores al utilizar alta señal. Se comparan tres métodos de medidas no lineales en piezocomposites donde la precisión de los resultados se incrementa en cada uno de ellos. Se analiza la respuesta de la corriente y de la velocidad de un punto del transductor en función de la amplitud de la excitación.
\end{abstract}

Palabras clave: Composites, No linealidad, Transductores, Ráfagas, END.

Nonlinear characterization with burst excitation of 1-3 piezocomposite power transducers for air emission

Non-Destructive Testing, as well as ultrasonic emission, frequently uses resonant transducers made with 1-3 piezocomposites. When the piezocomposite transducer is used for ultrasonic emission in air, it is necessary the use of high amplitude pulses to generate high acoustic signal. So, the nonlinear behavior must to be in account and the nonlinear measurements have been carried out with burst excitation in similar application conditions. The new proposed method measure current, as well as vibration velocity, of the piezocomposite transducer for burst signal excitation, near the thickness resonance frequency. The current of the stationary response is measured before the end of the burst, for different piezocomposite. Burst excitation enables us to measure the nonlinear behavior to avoid overheating the transducers, when high signals are used. Three piezocomposites nonlinear measurement methods are compared with an increase of the accuracy. The current and velocity dependence of a point of transducer versus the excitation of the amplitude level is analyzed.

Key words: Composites, Non linearity, Transducers, Burst, NDT.

\section{INTRODUCCIÓN}

Las ventajas que ofrecen los piezocomposites 1-3 en los transductores de potencia para la emisión de ultrasonidos en aire, hacen que su utilización en dispositivos de Ensayos No Destructivos o en general como emisores de ultrasonidos con transductores piezoeléctricos resonantes, sea cada vez mayor. Entre las ventajas destaca la posibilidad de reducir la impedancia acústica del transductor, logrando de esta forma una mejor adaptación de impedancias entre el resonador, el medio y el objeto a estudiar.

Utilizar transductores piezocomposites emitiendo ultrasonidos en aire implica el uso de grandes potencias para que la energía acústica emitida por el transductor sea significativa. Esto se logra con pulsos cortos cercanos a la frecuencia de resonancia de los composites que garanticen distinguir correctamente entre dos ecos recibidos, así la onda acústica emitida al medio nos proporciona una inspección completa de la pieza que nos interesa analizar (1). La señal a ráfagas o pulsada permite no solo una mayor excitación del transductor sino que evita el sobrecalentamiento de la muestra. Sin embargo, las deformaciones elevadas en el transductor generan comportamientos no lineales típicos de los dispositivos de potencia, por lo que, es necesario la caracterización del régimen no lineal.

Las medidas experimentales no lineales pueden realizarse utilizando varios métodos donde la señal de excitación puede ser estacionaria o pulsada. El método de variación de impedancias $(2,3)$ recurre a señales estacionarias (no burst) por el tipo de instrumentación que se utiliza, sin embargo, ya que no se desea un sobrecalentamiento del piezocomposite debe limitarse la potencia que se aplica al transductor. Otros métodos, utilizados en este trabajo utilizan una excitación en forma de ráfaga para presentar los resultados del comportamiento no lineal en dos zonas: en la cola de la excitación (régimen libre) $(4,5)$ y antes de finalizar la señal pulsada.

El nuevo método de caracterización no lineal que se propone mide la corriente y la velocidad de vibración del transductor justo antes del final del régimen de excitación. Las medidas no lineales realizadas se caracterizan por el estudio 
de la tangente de pérdidas mecánicas $\operatorname{tg} \delta_{m}$, de la variación del módulo de la rigidez $\left|\Delta c / c_{0}\right|$ y del cociente entre la velocidad y la corriente que circula a través de todo el transductor, siempre en función de la amplitud de la excitación y en condiciones próximas a las de las aplicaciones.

Este método permite un estudio preciso de las características no lineales a la vez que mejora la exactitud de los resultados al evitar las oscilaciones que se producen en la cola del régimen libre, provocadas por batidos entre vibraciones a diferentes frecuencias.

\section{CARACTERIZACIÓN NO LINEAL}

Las medidas de la no linealidad de los distintos composites, realizados en el Instituto de Acústica (CSIC) a partir de diferentes fracciones cerámicas de Ferroperm, se realizaron utilizando tres métodos de caracterización no lineal.

\subsection{Método de medida de variación de impedancias}

Este método se lleva a cabo haciendo un barrido de amplitudes crecientes a una frecuencia fija cercana a la resonancia. Se mide la impedancia de la muestra para cada valor de amplitud y la corriente que circula por el transductor con la ayuda de un Analizador de Impedancias/Redes/Espectros HP 4195A y una sonda de corriente TEK CT-2. El nivel máximo de excitación de las medidas de los composites esta limitado por condiciones netamente experimentales como es evitar el sobrecalentamiento del resonador debido a la excitación armónica. Este sistema de medidas no utiliza señales pulsadas y sólo se alcanzan potencias del orden de los 4 w sobre transductores de $7 \mathrm{~cm}^{2}$ de área.

\subsection{Método de medida a ráfagas (modo burst)}

La caracterización no lineal utilizando el método de medida a ráfagas permite una mayor excitación sin un incremento de la temperatura en la muestra. Esta ráfaga está formada por $\mathrm{N}$ ciclos que se repiten cada $10 \mathrm{~Hz}$ (figura 1). El número de ciclos y la frecuencia cercana a la de resonancia del composite proporciona un nivel importante de la respuesta mecánica. Las medidas no lineales se realizaron tanto en el régimen libre del resonador, como justo antes del final de la señal que se aplica. En ambas caracterizaciones la señal de la corriente se adquiere utilizando un osciloscopio digital Tektronix TDS 210.

\subsubsection{RÉGIMEN LIBRE.}

$\mathrm{Al}$ analizar la respuesta transitoria, la corriente medida, es producida por la vibración mecánica y obedece aproximadamente a una función exponencial decreciente (figura 1), donde las pérdidas mecánicas y la variación de la rigidez son magnitudes dependientes de la amplitud de la señal. La frecuencia de ésta respuesta transitoria es la frecuencia natural de las oscilaciones libres. Se asume que la respuesta transitoria de la carga libre en un circuito eléctrico resonante es:

$$
q(t)=A e^{-t / 2 \tau} \cos (\omega t),
$$

donde $\tau$ y $\omega$ son funciones de la amplitud, debido al comportamiento no lineal. Al considerar que la curva de la corriente
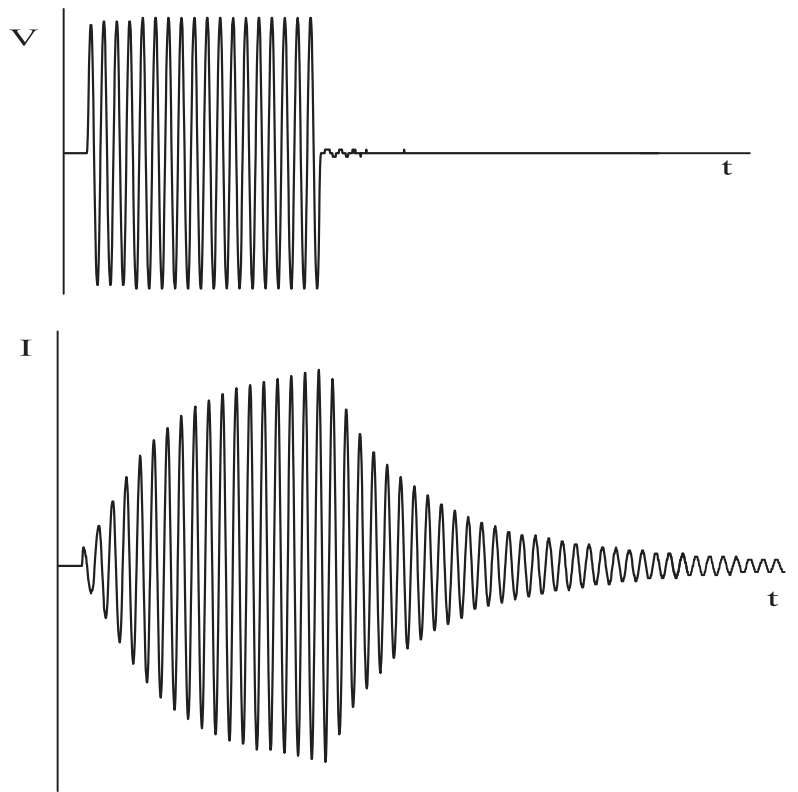

Figura 1: Excitación pulsada del voltaje y la corriente a través del transductor, donde se observa la respuesta transitoria después de finalizar la señal pulsada.

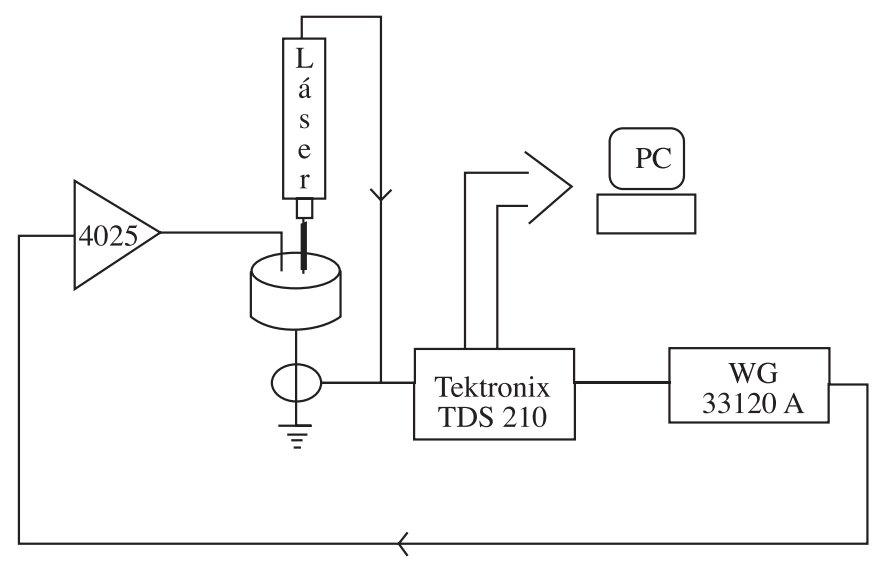

Figura 2: Sistema Experimental.

decrece suavemente, el tiempo de relajación del circuito $\tau$ puede ser medido a partir de dos máximos consecutivos $q_{a}(n)$ y $q_{a}(n+1)$, asumiendo que este tiempo de relajación es aproximadamente constante.

Por tanto, la tangente de pérdidas puede ser calculada por la expresión:

$$
\operatorname{tg} \delta_{m}=\frac{1}{Q_{m}}=\frac{1}{\omega \tau}=\frac{\ln q_{a}(n)-\ln q_{a}(n+1)}{\pi} .
$$

La medida del período de la oscilación permite calcular la dependencia de la frecuencia con la amplitud justo después del final de la excitación pulsada

Para obtener medidas con exactitud, la señal requiere un tratamiento completo que parte de la utilización de un filtro Hamming en la toma de datos del osciloscopio hasta la corrección de los valores de la tangente de pérdidas, considerando las pérdidas externas del sistema experimental, figura 2 . 
Este método (5) posibilita que las amplitudes de la señal de excitación se extiendan desde los 0.5 A hasta los 4 A, alcanzando potencias de $200 \mathrm{w}$ aproximadamente, 50 veces superior a las del método anterior sin que la temperatura del transductor aumente.

La figura 3 muestra la tangente de pérdidas no lineal en función de la deformación media para dos composites diferentes, uno blando, PZ 27 y otro duro, PZ 26 medida por los dos métodos. La concordancia que se aprecia entre métodos es buena.

Las pérdidas mecánicas de composites construidos con cerámicas blandas son mayores que las de composites con cerámicas duras. Se observa además que es posible alcanzar deformaciones elevadas con composites duros.

Sin embargo, el principal problema de este sistema de medida es que se producen batidos entre vibraciones a diferentes frecuencias, los cuales se relacionan con los distintos modos que aparecen en la respuesta transitoria, entre los que pueden incluirse el modo radial. Estos batidos se reflejan en las gráficas del comportamiento no lineal del régimen libre.

La velocidad $v(t)$, medida con un vibrómetro láser, de un punto del resonador muestra un comportamiento con mayores batidos que los observados en la función $I(t)$. La combinación de batidos en el régimen transitorio libre proporciona a las medidas no lineales un error que no permite un análisis riguroso de los piezocomposites.

Se hace necesario un nuevo método de medida donde se eviten los batidos y se aumente la exactitud de los resultados no lineales, sin renunciar a las altas potencias de la excitación a ráfagas.

\subsubsection{RÉGIMEN ESTACIONARIO.}

Las medidas se realizan en las últimas oscilaciones de la excitación a ráfagas antes que el resonador comience a oscilar por sí solo. El número de ciclos de cada ráfaga variará en función del tipo de composite, pues se necesita garantizar que la amplitud de la respuesta transitoria $I(t)$ sea prácticamente constante como corresponde al régimen estacionario.

El voltaje $V$ de la señal de excitación y la corriente $I$ de la respuesta del resonador se miden para cada nivel deseado, así como la diferencia de fase $\varphi$ entre ellas. Con la ayuda de un vibrómetro láser Polytec OFV 3001 y otro osciloscopio se calcula la relación entre la velocidad $v$ de un punto del material y la respuesta $I(t)$ de la señal que se aplica.

A partir de las medidas de $V$, I y el calculo de $\varphi$ es posible conocer el módulo de la impedancia $|Z|$, con lo cual, la parte real e imaginaria de $Z$ nos permite relacionar estas con la tangente de pérdidas mecánicas y la variación de la rigidez:

$$
\begin{gathered}
R=|Z| \cos (\varphi), \quad X=|Z| \operatorname{sen}(\varphi), \\
\operatorname{tg} \delta_{m}=\frac{R}{\bar{Z}}, \quad \frac{\Delta c}{c_{0}}=-\frac{2 \Delta f}{f_{s}}=\frac{\Delta X}{\bar{Z}}, \\
\bar{Z}=Q R_{1}, \quad Q=\frac{f_{s}}{\Delta f},
\end{gathered}
$$

donde, $R_{1}$ es la resistencia de la parte mecánica del circuito equivalente (5) y $Q$ el factor de calidad del piezocomposite.

La dependencia de la tangente de pérdidas y la variación relativa de la rigidez en función de la deformación media $\langle S\rangle$

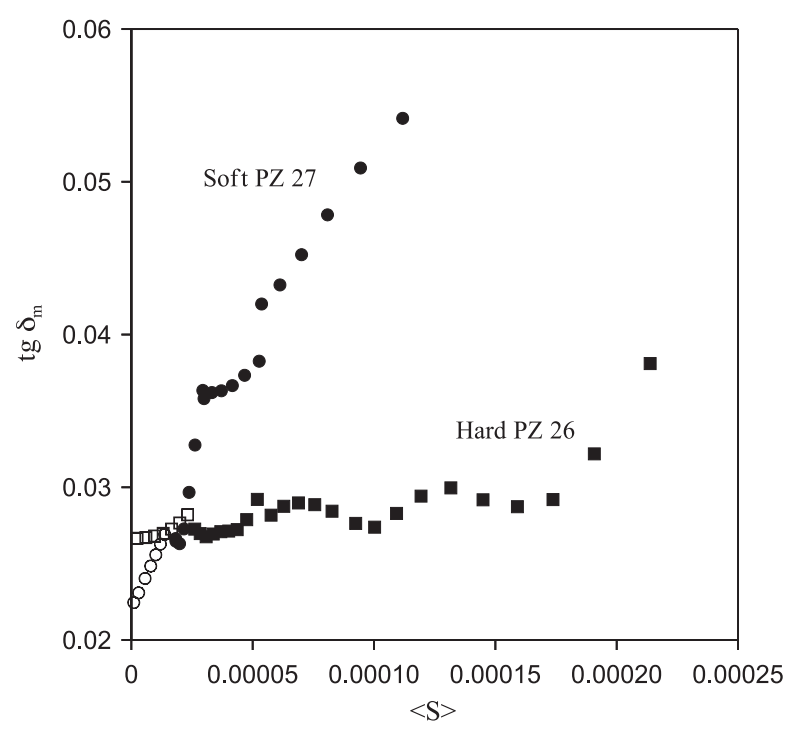

Figura 3: Tangente de pérdidas $\operatorname{tg} \delta_{m}$ en función de la deformación media $\langle$ S $>$ para composites blandos PZ $27(\bullet, 0)$ y duros PZ 26( $\square)$. Medidas pulsadas $(\bullet, \square)$ y de variación impedancia $(O, \square)$.

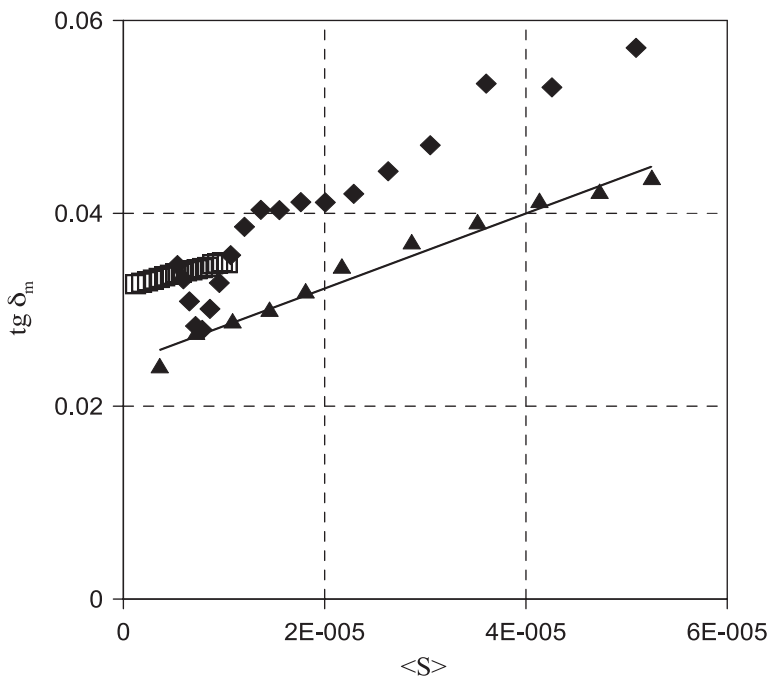

Figura 4: Dependencia de la tangente de pérdidas $\operatorname{tg} \delta_{m}$ en función de la deformación media $\langle S>$ para el PZ 27, $f=48 \%$. Método de variación de impedancias $\square$, Régimen Libre (burst) $\bullet$, Régimen Estacionario (burst) $\mathbf{\Delta}$.

se muestran en las figuras 4 y 5 . Analizar la correspondencia de estas variaciones no lineales con $\langle S>$ es importante, ya que la onda acústica producida por un transductor de potencia depende directamente de la amplitud de la deformación. La expresión que permite relacionar $<S>$ y $I$ es la siguiente (3):

$$
<>=\frac{I}{A \omega_{s} \sqrt{M}} \text {. }
$$

Siendo, $A$ la superficie de los electrodos, $w$ la frecuencia angular de la resonancia y $M$ el factor de mérito que para el modo grosor es:

$$
M_{33}=\mathrm{e}_{33}^{2} \text {. }
$$




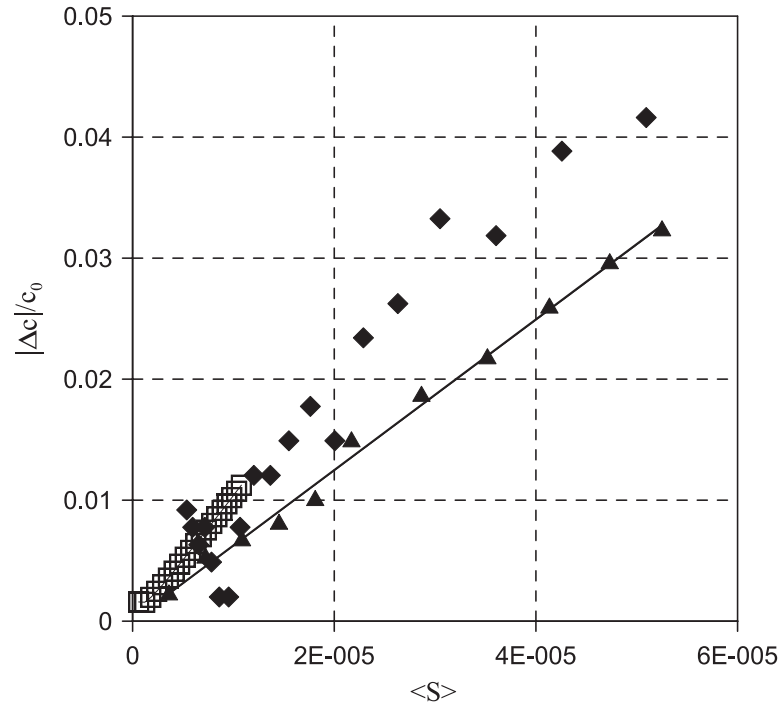

Figura 5: Dependencia del módulo de la variación de la rigidez $\left|\Delta c / c_{0}\right|$ en función de la deformación media $\langle S>$ para el PZ 27, $f=$ $48 \%$. Método de variación de impedancias $\square$, Régimen Libre (burst) $\bullet$, Régimen Estacionario (burst)

En este nuevo método las oscilaciones libres que aparecían después de finalizar la señal a ráfaga y que alteraban los resultados anteriores se han eliminado. Destacando una mejor linealidad en los resultados, así como concordancia con los métodos anteriores. Las altas señales se han mantenido alrededor de los $200 \mathrm{~W}$ sin que la temperatura del resonador aumente.

La curva del cociente entre la velocidad de un punto del transductor y la corriente que circula por él en función de la amplitud de la excitación muestra que la proporcionalidad entre estas magnitudes es constante, incluso con alta señal (figura 6). Esto permite considerar el factor de mérito constante. La velocidad $v(t)$ no es una magnitud representativa de toda la muestra sino de cada punto en específico como consecuencia de la presencia de batidos a diferentes frecuencias.

\subsection{Comparación entre métodos de caracterización no lineal.}

La tabla I muestra las características principales de cada uno de los métodos de caracterización no lineal utilizados. En ella puede apreciarse de forma clara sus ventajas e inconvenientes.

\section{CONCLUSIONES}

Se comparan tres métodos de caracterización no lineal donde el uso de señales a ráfagas en dos ellos, permite llegar a amplitudes mucho más grandes que utilizando el método de variación de impedancias. Utilizar señales a ráfagas condiciona que la temperatura del transductor no aumente manteniendo los niveles de alta señal, garantizando la caracterización no lineal. Se analizan las ventajas e inconvenientes de cada uno de ellos.

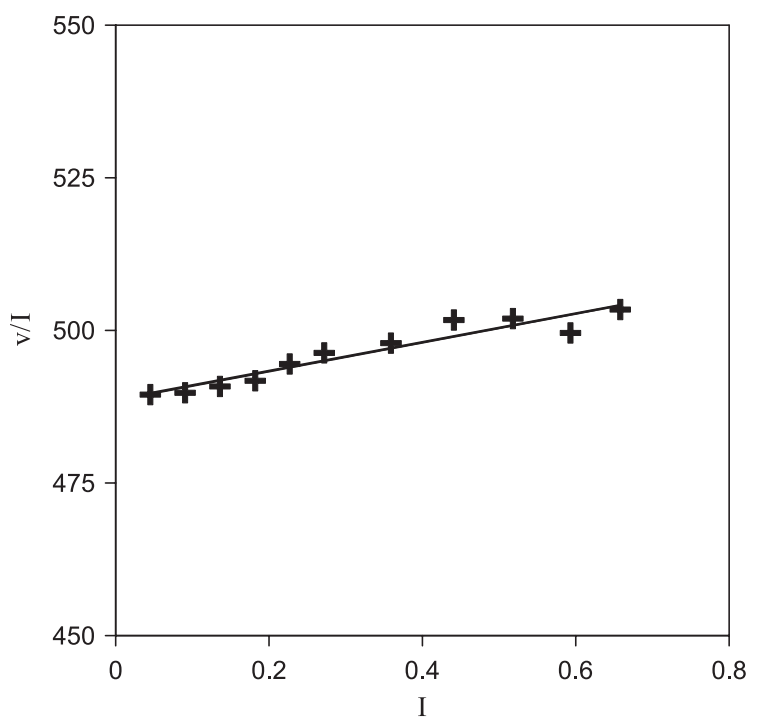

Figura 6: Cociente de la velocidad de un punto del resonador y la corriente que circula por él en función de la amplitud de la excitación.

Tabla I: MÉtodos de Caracterización No Lineal.

\begin{tabular}{|l|l|l|}
\hline Mét. de medida No Lineal & \multicolumn{1}{|c|}{ Ventajas } & \multicolumn{1}{c|}{ Inconvenientes } \\
\hline Variación de Impedancias & $\begin{array}{l}\text { - Medidas precisas } \\
\text { Instrumentación precisa }\end{array}$ & $\bullet$ Potencia máxima pequeña \\
\hline Reg. Libre Ráfaga & $\begin{array}{l}\text { - Potencia máxima elevada } \\
\text { Medidas rápidas (todos los } \\
\text { puntos en 1 medida) }\end{array}$ & $\begin{array}{l}\text { Poca precisión debido a } \\
\text { batidos }\end{array}$ \\
\hline Reg. Estacionario Ráfaga & $\begin{array}{l}\text { - Potencia máxima elevada } \\
\text { Buena precisión de } \\
\text { medidas }\end{array}$ & $\begin{array}{l}\bullet \text { Medidas más lentas } \\
\text { (1 punto por medida) }\end{array}$ \\
\hline
\end{tabular}

El uso del régimen libre para la caracterización no lineal de materiales piezoeléctricos facilita resultados correctos, siempre que se trabaje en el primer modo, es decir el de frecuencia más baja (4). En otras circunstancias, como la que el presente trabajo estudia, de un piezocomposite en modo grosor, en el régimen libre aparecen diferentes modos posibles dando lugar a batidos que se superponen al decrecimiento exponencial de este régimen, haciendo que los resultados no lineales obtenidos estén alterados y con un error muy grande.

En contraposición el método aquí propuesto que es totalmente original y novedoso utiliza las virtudes de una excitación a ráfagas pero evita los inconvenientes del régimen libre, al realizar las medidas en la zona de la ráfaga antes de su final. Se impone al transductor la frecuencia de la oscilación y se evitan las oscilaciones libres de los diferentes modos propios.

Como se muestra en este trabajo, este nuevo método ofrece resultados acordes con los anteriores métodos pero con una mejor precisión que el método que utiliza el régimen libre y aplicando potencias más elevadas que las utilizadas en el método de variación de impedancias. 


\section{AGRADECIMIENTOS}

Este trabajo ha sido posible gracias a la ayuda de la CICYT, proyectos nº TAP98-0911-C03-02 y TAP98-0911-C03-01

\section{BIBLIOGRAFÍA}

1. J. A. Casals, "Caracterización No Lineal de Piezocomposites Resonantes en Régimen Estacionario e Impulsional. Proyecto de Tesis Doctoral", Actes de les Jornades de Doctorat, Departaments de Física Aplicada i de Física i Enginyeria Nuclear. Julio, 2000.
2. R. Pérez, A. Albareda, "Analysis of Nonlinear Effects in a Piezoelectric Resonator", J. Acous. Soc. Am. 100 [6] 3561-3570 (1996)

3. J. A. Casals, A. Albareda, R. Pérez, J. H. Kayombo, E. Minguella, F. Montero de Espinosa, "Nonlinear Characterization of Resonant Piezocomposites 1-3 for NDT Ultrasonic Transducers", The European Physical Journal-Applied Physics 11 15-20 (2000)

4. S. Takahashi, Y. Sasaki, M. Umeda, K. Nakamura, S. Ueha, "Nonlinear Behavior in Piezoelectric Ceramic Transducers", Proceedings of the $200012^{\text {th }}$ IEEE-ISAF, Hawaii (U.S.A.) July 31-August 22000 IEEE.

5. A. Albareda, J. A. Casals R. Pérez, F. Montero de Espinosa, "Nonlinear Measurements of Piezocomposite Transducers with Burst Excitation", Proceedings of the $200012^{\text {th }}$ IEEE-ISAF, Hawaii (U.S.A.) July 31-August 2 2000 IEEE.

Recibido: 31.05.01

Aceptado: 10.12 .01 